\title{
Cohomological non-rigidity of eight-dimensional complex projective towers
}

\author{
SHINTARÔ KUROKI \\ DONG YOUP SUH
}

\begin{abstract}
A complex projective tower, or simply $\mathbb{C P}$ tower, is an iterated complex projective fibration starting from a point. In this paper, we classify a certain class of 8 -dimensional $\mathbb{C P}$ towers up to diffeomorphism. As a consequence, we show that cohomological rigidity is not satisfied by the collection of 8 -dimensional $\mathbb{C P}$ towers: there are two distinct 8 -dimensional $\mathbb{C P}$ towers that have the same cohomology rings.
\end{abstract}

57R22; 57S25

\section{Introduction}

Let $\mathcal{M}$ be a collection of diffeomorphism classes of smooth manifolds, and let $\boldsymbol{H}^{*} \mathcal{M}$ be the isomorphism classes of cohomology rings of manifolds in $\mathcal{M}$. Let $H^{*}: \mathcal{M} \rightarrow \boldsymbol{H}^{*} \mathcal{M}$ be the map defined by $M \in \mathcal{M} \mapsto H^{*}(M ; \mathbb{Z})$. In general, $H^{*}$ is not bijective. However, if we restrict the class of manifolds then this map sometimes becomes a bijection. For example, if $\mathcal{M}$ is a collection of orientable 2-dimensional manifolds then it is well known that the map $H^{*}$ is bijective. We say such a collection $\mathcal{M}$ is cohomologically rigid, or that $\mathcal{M}$ satisfies cohomological rigidity. The problem of whether the map $H^{*}: \mathcal{M} \rightarrow \boldsymbol{H}^{*} \mathcal{M}$ is bijective or not is called the cohomological rigidity problem. In this paper, we study the cohomological rigidity problem for complex projective towers (or simply $\mathbb{C P}$ towers), which we introduced in [7].

A $\mathbb{C P}$ tower of height $m$ is a sequence of complex projective fibrations

$$
C_{m} \stackrel{\pi_{m}}{\longrightarrow} C_{m-1} \stackrel{\pi_{m-1}}{\longrightarrow} \cdots \stackrel{\pi_{2}}{\longrightarrow} C_{1} \stackrel{\pi_{1}}{\longrightarrow} C_{0}=\{\text { point }\}
$$

where $C_{i}=P\left(\xi_{i-1}\right)$ is the projectivization of a complex vector bundle $\xi_{i-1}$ over $C_{i-1}$. We call each $C_{i}$ the $i^{\text {th }}$ stage of the tower. If we forget the tower structure, then we call $C_{i}$ an ( $i$-stage) $\mathbb{C P}$ manifold. In [7], we show that the diffeomorphism types of 6-dimensional $\mathbb{C P}$ manifolds are determined by their cohomology rings; ie the collection of 6 -dimensional $\mathbb{C P}$ manifolds $\mathbb{C P} \mathcal{M}^{6}$ is cohomologically rigid. This is a generalization of the fact, due to Choi, Masuda and Suh [5], that the collection $\mathcal{G B M}^{6}$ 
of 6-dimensional generalized Bott manifolds is cohomologically rigid. It is also known that the collection $\mathcal{G B M}_{2}^{2 n}$ of $2 n$-dimensional 2-stage generalized Bott manifolds is cohomologically rigid. The purpose of this paper is to show that the collection $\mathbb{C P M}_{2}^{8}$ of 8-dimensional 2-stage $\mathbb{C P}$ manifolds is not cohomologically rigid.

To state our main theorem, let us recall a theorem of Atiyah and Rees [1, Theorem 2.8]. Let $\operatorname{Vect}_{2}\left(\mathbb{C P}^{3}\right)$ be the collection of isomorphism classes of 2-dimensional complex vector bundles over $\mathbb{C P}^{3}$.

Theorem 1.1 (Atiyah-Rees) There exists an injective map

$$
\phi: \operatorname{Vect}_{2}\left(\mathbb{C P}^{3}\right) \rightarrow \mathbb{Z}_{2} \oplus \mathbb{Z} \oplus \mathbb{Z}, \quad \xi \mapsto\left(\alpha(\xi), c_{1}(\xi), c_{2}(\xi)\right),
$$

where $c_{1}(\xi)$ and $c_{2}(\xi)$ are the first and the second Chern classes of $\xi$, and $\alpha(\xi) \in \mathbb{Z}_{2}$ is 0 when $c_{1}(\xi)$ is odd.

By Theorem 1.1, any element in $\operatorname{Vect}_{2}\left(\mathbb{C P}^{3}\right)$ can be denoted by $\eta_{\left(\alpha, c_{1}, c_{2}\right)}$, where $\left(\alpha, c_{1}, c_{2}\right) \in \mathbb{Z}_{2} \oplus \mathbb{Z} \oplus \mathbb{Z}$ is such that $\alpha \equiv 0(\bmod 2)$ when $c_{1} \equiv 1(\bmod 2)$. On the other hand, it's easy to see that $P\left(\eta_{\left(\alpha, c_{1}, c_{2}\right)}\right)$ is diffeomorphic to $P\left(\eta_{\left(0,1, c_{2}-\left(c_{1}^{2}-1\right) / 4\right)}\right)$ if $c_{1} \equiv 1 \bmod 2$, and diffeomorphic to $P\left(\eta_{\left(\alpha, 0, c_{2}-c_{1}^{2} / 4\right)}\right)$ if $c_{1} \equiv 0 \bmod 2$; see Lemma 3.2.

We now state the main result of the paper; see Theorem 4.2 for (1) and Theorem 5.2 for a more precise statement of (2).

Theorem 1.2 Let $N(u):=P\left(\eta_{(0,1, u)}\right)$ and $\mathcal{N}:=\{N(u) \mid u \in \mathbb{Z}\}$. Similarly, let $M_{\alpha}(u):=P\left(\eta_{(\alpha, 0, u)}\right)$ and $\mathcal{M}:=\left\{M_{\alpha}(u) \mid \alpha \in\{0,1\}, u \in \mathbb{Z}\right\}$.

(1) $\mathcal{N}$ is cohomologically rigid. In fact, the following are equivalent:

(a) $N(u)$ is diffeomorphic to $N\left(u^{\prime}\right)$.

(b) $u=u^{\prime}$.

(c) $H^{*}(N(u) ; \mathbb{Z})$ and $H^{*}\left(N\left(u^{\prime}\right) ; \mathbb{Z}\right)$ are isomorphic as graded rings.

(2) $\mathcal{M}$ is not cohomologically rigid. In fact, $H^{*}\left(M_{0}(u) ; \mathbb{Z}\right)$ and $H^{*}\left(M_{1}(u) ; \mathbb{Z}\right)$ are isomorphic as graded rings for all $u$, but if $u(u+1) / 12 \in \mathbb{Z}$ then $M_{0}(u)$ is not diffeomorphic, or even homotopic, to $M_{1}(u)$.

We prove (2) in Proposition 5.4 by showing that $\pi_{6}\left(M_{0}(u)\right) \not \pi_{6}\left(M_{1}(u)\right)$ when $u(u+1) / 12 \in \mathbb{Z}$.

The organization of this paper is as follows. In Section 2, as examples of $\mathbb{C P}$ towers, we explain when a flag manifold admits the structure of a $\mathbb{C P}$ tower. In Section 3, we recall some basic facts from [7]. In Section 4, we show that $\mathcal{N}$ satisfies cohomological rigidity. In Section 5, we compute the 6-dimensional homotopy group of the elements in some class of $\mathcal{M}$ and show that $\mathcal{M}$ does not satisfy cohomological rigidity. 
Acknowledgements The authors would like to thank the referee for invaluable comments. In particular, the proofs of Lemma 3.3 and Lemma 4.1 are greatly simplified by their suggestions. The first author would like to give heartfelt thanks to Professor Nigel Ray, whose comments and help at the University of Manchester were immeasurably valuable. He also would like to thank to Professor Yael Karshon of the University of Toronto and Professor Takashi Tsuboi of the University of Tokyo for excellent circumstances to do research. Finally, the first author was partially supported by Grantin-Aid for Scientific Research (S) number 24224002 of the Japan Society for Promotion of Science, the JSPS Institutional Program for Young Researcher Overseas Visits "promoting international young researchers in mathematics and mathematical sciences led by OCAMI", and the JSPS Strategic Young Researcher Overseas Visits Program for Accelerating Brain Circulation "Deepening and Evolution of Mathematics and Physics, Building of International Network Hub based on OCAMI". The second author was supported in part by the Basic Science Research Program through the National Research Foundation of Korea (NRF) funded by the Ministry of Education (grant number 2013R1A1A2007780).

\section{Flag manifolds of type A and C}

$\mathbb{C P}$ towers include many interesting classes of manifolds. In a previous paper [7], we showed that generalized Bott manifolds and the Milnor hypersurface admit a $\mathbb{C P}$ tower structure. We first introduce two other examples of $\mathbb{C P}$ towers. Let $\mathbb{C P} \mathcal{M}_{m}^{2 n}$ be the collection of $2 n$-dimensional $m$-stage $\mathbb{C P}$ manifolds up to diffeomorphism.

Example 2.1 A partial flag manifold $\mathcal{F}\left(d_{1}, d_{2}, \ldots, d_{k}\right)$, where $0=d_{0}<d_{1}<d_{2}<$ $\cdots<d_{k-1}<d_{k}=n+1$, is defined by the set of partial flags

$$
\{0\} \subset V_{1} \subset V_{2} \subset \cdots \subset V_{k-1} \subset V_{k}=\mathbb{C}^{n+1},
$$

where $V_{i}$ is a complex subspace of $\mathbb{C}^{n+1}$ of complex dimension $d_{i}$. This is well known to be diffeomorphic to the homogeneous space $U(n+1) /\left(U\left(n_{1}\right) \times \cdots \times U\left(n_{k}\right)\right)$, where $n_{i}=d_{i}-d_{i-1}$ for $i=1, \ldots, k$. Denote the partial flag manifold $\mathcal{F}(i, i+1$, $\ldots, n+1)$ by $\mathcal{F}_{i}$. In particular, we call $\mathcal{F}_{1}=\mathcal{F}(1,2, \ldots, n+1)$ a flag manifold of type A (or a complete flag manifold), and denote it by $\mathcal{F l}\left(\mathbb{C}^{n+1}\right)$. We will show that the flag manifold of type A has the structure of a $\mathbb{C P}$ tower with height $n$. We first define a map $p_{i}: \mathcal{F}_{i} \rightarrow \mathcal{F}_{i+1}$ by

$$
p_{i}:\{0\} \subset V_{i} \subset V_{i+1} \subset \cdots \subset V_{n} \subset \mathbb{C}^{n+1} \mapsto\{0\} \subset V_{i+1} \subset \cdots \subset V_{n} \subset \mathbb{C}^{n+1} .
$$

As the pull-back of a point in $\mathcal{F}_{i+1}$ by $p_{i}$ can be regarded as the set of codimensionone subspaces $V_{i} \subset V_{i+1}, \mathcal{F}_{i}$ is a $\operatorname{Gr}_{i}\left(V_{i+1}\right)$-bundle over $\mathcal{F}_{i+1}$. Here, $\operatorname{Gr}_{i}\left(V_{i+1}\right)$ 
is the complex Grassmaniann of $i$-dimensional subspaces in $V_{i+1}$; ie $\mathcal{F}(i, i+1)$. Because the normal subspace of a codimension-one subspace $V_{i} \subset V_{i+1}$ is just a line through the origin, the complex Grassmaniann $\operatorname{Gr}_{i}\left(V_{i+1}\right)$ may be regarded as the $i$-dimensional complex projective space $\mathbb{C P}\left(V_{i+1}\right)=\left(V_{i+1} \backslash\{0\}\right) / \mathbb{C}^{*}$. Using this fact, it is easy to check that $\mathcal{F}_{i}$ is the projectivization of the tautological bundle over $\mathcal{F}_{i+1}$; ie $\mathcal{F}_{i}=\mathbb{C P}\left(\eta_{i+1}\right)$, where the tautological bundle $\eta_{i+1}$ is the complex $(i+1)$-dimensional vector bundle defined by the subset

$$
\left\{\left(\{0\} \subset V_{i+1} \subset \cdots \subset V_{n} \subset \mathbb{C}^{n+1}, x\right) \mid x \in V_{i+1}\right\}
$$

of $\mathcal{F}_{i+1} \times \mathbb{C}^{n+1}$. Therefore, $\mathcal{F l}\left(\mathbb{C}^{n+1}\right)$ has the structure of a $\mathbb{C P}$ tower:

$$
\mathcal{F} l\left(\mathbb{C}^{n+1}\right)=P\left(\eta_{2}\right) \stackrel{\mathbb{C P}^{1}}{\longrightarrow} \mathcal{F}_{2}=P\left(\eta_{3}\right) \stackrel{\mathrm{CP}^{2}}{\longrightarrow} \cdots \stackrel{\mathbb{C} \mathrm{P}^{n-1}}{\longrightarrow} \mathcal{F}_{n} \simeq \mathbb{C P}^{n} \longrightarrow\{*\} .
$$

Hence the flag manifold of type $\mathrm{A}$ is an element of $\mathrm{CPM}_{n}^{n^{2}+n}$.

Example 2.2 Let $\left(\mathbb{C}^{2 n}, \omega\right)$ be a complex vector space with a symplectic structure $\omega$ given by the skew-symmetric bilinear form

$$
\Omega=\left(\begin{array}{cc}
O & I_{n} \\
-I_{n} & O
\end{array}\right)
$$

where $O$ is the $n \times n$ zero matrix and $I_{n}$ is the $n \times n$ identity matrix. Let $V$ be a complex linear subspace in $\mathbb{C}^{2 n}$. Define the $\omega$-perpendicular space of $V$ to be the subspace

$$
V^{\omega}=\left\{w \in \mathbb{C}^{2 n} \mid \omega(v, w)=v^{T} \Omega w=0 \text { for all } v \in V\right\} .
$$

Note that $\left(V^{\omega}\right)^{\omega}=V$ and $\operatorname{dim}_{\mathbb{C}} V+\operatorname{dim}_{\mathbb{C}} V^{\omega}=2 n$. We call $V$ isotropic or coisotropic if $V \subset V^{\omega}$ or $V^{\omega} \subset V$, respectively. A symplectic partial flag manifold $\operatorname{Sp}^{n} \mathcal{F}\left(d_{1}, d_{2}, \ldots, d_{k}\right)$, where $0=d_{0}<d_{1}<d_{2}<\cdots<d_{k-1}<d_{k} \leq n$, is defined by the set of (isotropic) partial flags

$$
\{0\} \subset V_{1} \subset V_{2} \subset \cdots \subset V_{k-1} \subset V_{k} \subset \mathbb{C}^{2 n},
$$

where $V_{i}$ is a complex isotropic subspace of $\left(\mathbb{C}^{2 n}, \omega\right)$ of complex dimension $d_{i}$. It is easy to check that this is equivalent to the set of partial flags

$$
\{0\} \subset V_{1} \subset \cdots \subset V_{k-1} \subset V_{k} \subset V_{k}^{\omega} \subset V_{k-1}^{\omega} \subset \cdots \subset V_{1}^{\omega} \subset \mathbb{C}^{2 n} .
$$

This is well known to be diffeomorphic to the homogeneous space $\operatorname{Sp}(n) /\left(U\left(n_{1}\right) \times \cdots \times\right.$ $\left.U\left(n_{k}\right) \times \operatorname{Sp}\left(n_{k+1}\right)\right)$, where $n_{i}=d_{i}-d_{i-1}$ for $i=1, \ldots, k$ and $n_{k+1}=\frac{1}{2}\left(\operatorname{dim} V_{k}^{\omega}-\right.$ $\left.\operatorname{dim} V_{k}\right)=n-d_{k}$. If $d_{k}=\frac{1}{2} \operatorname{dim} V_{k}=n$, ie $V_{k}=V_{k}^{\omega}$ is a Lagrangian subspace, then $\operatorname{Sp}^{n} \mathcal{F}\left(d_{1}, d_{2}, \ldots, d_{k-1}, n\right)$ is diffeomorphic to $\operatorname{Sp}(n) /\left(U\left(n_{1}\right) \times \cdots \times U\left(n_{k}\right)\right)$. Denote the symplectic partial flag manifold $\operatorname{Sp}^{n} \mathcal{F}(1,2, \ldots, i)$ by $\operatorname{Sp}^{n} \mathcal{F}_{i}$ for $i \geq 1$. 
In particular, we call $\mathrm{Sp}^{n} \mathcal{F}_{n}=\operatorname{Sp}^{n} \mathcal{F}(1,2, \ldots, n)$ a flag manifold of type $C$ (or a symplectic flag manifold), and denote it by $\operatorname{Sp} \mathcal{F} l\left(\mathbb{C}^{2 n}\right)$. We will show that the flag manifold of type $\mathrm{C}$ has the structure of a $\mathbb{C P}$ tower with height $n$. We first define a map $q_{i}: \operatorname{Sp}^{n} \mathcal{F}_{i+1} \rightarrow \operatorname{Sp}^{n} \mathcal{F}_{i}$ by

$$
\begin{aligned}
q_{i}:\{0\} \subset V_{1} \subset \cdots \subset V_{i} \subset V_{i+1} \subset & V_{i+1}^{\omega} \subset V_{i}^{\omega} \subset \cdots \subset V_{1}^{\omega} \subset \mathbb{C}^{2 n} \\
& \mapsto\{0\} \subset V_{1} \subset \cdots \subset V_{i} \subset V_{i}^{\omega} \subset \cdots \subset V_{1}^{\omega} \subset \mathbb{C}^{2 n} .
\end{aligned}
$$

The pull-back of a point in $\operatorname{Sp}^{n} \mathcal{F}_{i}$ by $q_{i}$ can be regarded as the set of isotropic subspaces $V_{i+1}$ in $\mathbb{C}^{2 n}$ which contain the isotropic subspace $V_{i}$ as a codimension-one subspace. Note that for any vectors $v \in V_{i}^{\omega} \backslash V_{i}$, the subspace $V_{i} \oplus \operatorname{span}_{\mathbb{C}}(v)$ is an isotropic subspace which contains $V_{i}$ as a codimension-one subspace. Therefore, there exists a one-to-one correspondence between the pull-back of a point in $\operatorname{Sp}^{n} \mathcal{F}_{i}$ by $q_{i}$ and all complex lines in the quotient vector space $V_{i}^{\omega} / V_{i} \simeq \mathbb{C}^{2 n-2 i}$; ie $\mathrm{Sp}^{n} \mathcal{F}_{i+1}$ is a $\mathbb{C P}^{2 n-2 i-1}$-bundle over $\operatorname{Sp}^{n} \mathcal{F}_{i}$. Using this fact, it is easy to check that $\operatorname{Sp}^{n} \mathcal{F}_{i+1}$ is the projectivization of the quotient bundle over $\mathrm{Sp}^{n} \mathcal{F}_{i}$; ie $\mathrm{Sp}^{n} \mathcal{F}_{i+1}=P\left(\zeta_{i}^{\omega} / \zeta_{i}\right)$, where the two tautological bundles $\zeta_{i}^{\omega}$ and $\zeta_{i}$ are defined by the following subsets in $\operatorname{Sp}^{n} \mathcal{F}_{i} \times \mathbb{C}^{2 n}$, respectively:

$$
\begin{gathered}
\left\{\left(\{0\} \subset V_{1} \subset \cdots \subset V_{i} \subset V_{i}^{\omega} \subset \cdots \subset V_{1}^{\omega} \subset \mathbb{C}^{2 n}, x\right) \mid x \in V_{i}^{\omega}\right\}, \\
\left\{\left(\{0\} \subset V_{1} \subset \cdots \subset V_{i} \subset V_{i}^{\omega} \subset \cdots \subset V_{1}^{\omega} \subset \mathbb{C}^{2 n}, x\right) \mid x \in V_{i}\right\} .
\end{gathered}
$$

Note that $\zeta_{i}^{\omega}$ is a $\mathbb{C}^{2 n-i}$-vector bundle and $\zeta_{i}$ is a $\mathbb{C}^{i}$-vector bundle; therefore, the quotient bundle $\zeta_{i}^{\omega} / \zeta_{i}$ is a $\mathbb{C}^{2 n-2 i}$-vector bundle. Therefore, $\operatorname{Sp} \mathcal{F} l\left(\mathbb{C}^{2 n}\right)$ has the structure of a $\mathbb{C P}$ tower:

$$
\begin{aligned}
\operatorname{Sp} \mathcal{F} l\left(\mathbb{C}^{2 n}\right)=P\left(\zeta_{n-1}^{\omega} / \zeta_{n-1}\right) \stackrel{\mathbb{C P}{ }^{1}}{\longrightarrow} \operatorname{Sp}^{n} \mathcal{F}_{n-1}=P\left(\zeta_{n-2}^{\omega} / \zeta_{n-2}\right) & \stackrel{\mathbb{C P}^{3}}{\longrightarrow} \cdots \stackrel{\mathbb{C P}^{2 n-3}}{\longrightarrow} \operatorname{Sp}^{n} \mathcal{F}_{1} \\
& \simeq \mathbb{C} P^{2 n-1} \longrightarrow\{*\}
\end{aligned}
$$

Hence the flag manifold of type $\mathrm{C}$ is an element of $\mathrm{CPM}_{n}^{2 n^{2}}$.

Remark 1 As is well known, both of the flag manifolds $\mathcal{F} l\left(\mathbb{C}^{n+1}\right) \simeq U(n+1) / T^{n+1}$ and $\operatorname{Sp} \mathcal{F} l\left(\mathbb{C}^{2 n}\right) \simeq \operatorname{Sp}(n) / T^{n}$ with $n \geq 2$ do not admit the structure of a toric manifold; see [3], for example. On the other hand, $U(2) / T^{2} \cong \operatorname{Sp}(1) / T^{1} \cong \mathbb{C P}^{1}$ is a toric manifold.

Moreover, by computing the generators of flag manifolds of other types $-B_{n}(n \geq 3)$, $D_{n}(n \geq 4), G_{2}, F_{4}, E_{6}, E_{7}, E_{8}$ - we see that not all flag manifolds admit the structure of a $\mathbb{C P}$ tower; see [2], or [6] for classical types. This leads us to the following proposition. 
Proposition 2.3 Let $M=G / T$ be a flag manifold, where $G$ is a compact simple Lie group and $T$ is its maximal torus. If $M$ admits the structure of a $\mathbb{C P}$ tower, then $G$ must be a compact Lie group of type $A$ or $C$.

The following open problem naturally arises (also see Remark 2).

Problem 1 Let $H^{*}: \mathbb{C P} \mathcal{M} \rightarrow \boldsymbol{H}^{*} \mathbb{C P} \mathcal{M}$ be the map defined by taking cohomology rings. Classify the diffeomorphism types of all manifolds in the classes

$$
\left(H^{*}\right)^{-1}\left(H^{*}\left(U(n+1) / T^{n+1}\right)\right) \text { and }\left(H^{*}\right)^{-1}\left(H^{*}\left(\operatorname{Sp}(n) / T^{n}\right)\right) .
$$

\section{Some preliminaries}

\section{A Preliminaries from [7]}

We first recall some basic facts from [7, Section 2].

Let $\xi$ be an $n$-dimensional complex vector bundle over a topological space $X$, and let $P(\xi)$ denote its projectivization. Then

$$
H^{*}(P(\xi) ; \mathbb{Z}) \cong H^{*}(X ; \mathbb{Z})[x] /\left\langle x^{n+1}+\sum_{i=1}^{n}(-1)^{i} c_{i}\left(\pi^{*} \xi\right) x^{n+1-i}\right\rangle,
$$

where $\pi^{*} \xi$ is the pull-back of $\xi$ along $\pi: P(\xi) \rightarrow X$ and $c_{i}\left(\pi^{*} \xi\right)$ is the $i^{\text {th }}$ Chern class of $\pi^{*} \xi$ [7]. Here $x$ can be viewed as the first Chern class of the canonical line bundle over $P(\xi)$; ie the complex 1-dimensional sub-bundle $\gamma_{\xi}$ in $\pi^{*} \xi \rightarrow P(\xi)$ such that the restriction $\left.\gamma_{\xi}\right|_{\pi^{-1}(a)}$ is the canonical line bundle over $\pi^{-1}(a) \cong \mathbb{C P}^{n-1}$ for all $a \in X$. Therefore $\operatorname{deg} x=2$. Since it is well known that the induced homomorphism $\pi^{*}: H^{*}(X ; \mathbb{Z}) \rightarrow H^{*}(P(\xi) ; \mathbb{Z})$ is injective, we often abuse the notation $c_{i}\left(\pi^{*} \xi\right)$ by writing $c_{i}(\xi)$. The formula (2) is called the Borel-Hirzebruch formula.

To prove the main theorem, we often use the following two lemmas.

Lemma 3.1 Let $\gamma$ be any complex line bundle over $M$ and let $P(\xi)$ be the projectivization of a complex vector bundle $\xi$ over $M$. Then $P(\xi)$ is diffeomorphic to $P(\xi \otimes \gamma)$.

Lemma 3.2 Let $\gamma$ be a complex line bundle and let $\xi$ be a 2-dimensional complex vector bundle over a manifold $M$. Then the Chern classes of the tensor product $\xi \otimes \gamma$ are

$$
\begin{aligned}
& c_{1}(\xi \otimes \gamma)=c_{1}(\xi)+2 c_{1}(\gamma), \\
& c_{2}(\xi \otimes \gamma)=c_{1}(\gamma)^{2}+c_{1}(\gamma) c_{1}(\xi)+c_{2}(\xi) .
\end{aligned}
$$




\section{B The Atiyah-Rees theorem}

By Theorem 1.1, all of the complex 2-plane bundles over $\mathbb{C P}^{3}$ can be written $\eta_{\left(\alpha, c_{1}, c_{2}\right)}$ for some $\left(\alpha, c_{1}, c_{2}\right) \in \mathbb{Z}_{2} \times \mathbb{Z} \times \mathbb{Z}$. Using Lemma 3.1, its projectivization $P\left(\eta_{\left(\alpha, c_{1}, c_{2}\right)}\right)$ is diffeomorphic to $P\left(\eta_{\left(\alpha, c_{1}, c_{2}\right)} \otimes \gamma\right)$ for any complex line bundle $\gamma$ over $\mathbb{C P}^{3}$. By Lemma 3.2 and the proof of [1, Theorem 2.8] (Theorem 1.1 here), we also have

$$
\eta_{\left(\alpha, c_{1}, c_{2}\right)} \otimes \gamma \equiv \eta_{\left(\alpha, c_{1}+2 c_{1}(\gamma), c_{1}(\gamma)^{2}+c_{1}(\gamma) c_{1}+c_{2}\right)} .
$$

Thus we may assume $c_{1} \in\{0,1\}$. Consequently, to classify all $P\left(\eta_{\left(\alpha, c_{1}, c_{2}\right)}\right)$ up to diffeomorphisms, it is enough to classify

$$
\begin{aligned}
M_{0}(u) & =P\left(\eta_{(0,0, u)}\right), \\
M_{1}(u) & =P\left(\eta_{(1,0, u)}\right), \\
N(u) & =P\left(\eta_{(0,1, u)}\right),
\end{aligned}
$$

with $u \in \mathbb{Z}$. We denote the class of $M_{0}(u), M_{1}(u)$ up to diffeomorphism by $\mathcal{M}$ and that of $N(u)$ by $\mathcal{N}$. Then both classes $\mathcal{M}$ and $\mathcal{N}$ are subclasses of $\mathbb{C P} \mathcal{M}_{2}^{8}$ consisting of 8-dimensional 2-stage $\mathbb{C P}$ manifolds.

\section{C The intersection of $\mathcal{M}$ and $\mathcal{N}$ is empty}

We prove that $\mathcal{M} \cap \mathcal{N}=\varnothing$ by comparing cohomology rings. Namely, we prove the following lemma.

Lemma 3.3 Two cohomology rings $H^{*}\left(M_{\alpha}(u)\right)$ and $H^{*}\left(N\left(u^{\prime}\right)\right)$ are not isomorphic for any $u, u^{\prime} \in \mathbb{Z}$.

Proof Using the Borel-Hirzebruch formula (2), we have the cohomology rings with $\mathbb{Z}_{2}$-coefficients

$$
\begin{aligned}
H^{*}\left(M_{\alpha}(u) ; \mathbb{Z}_{2}\right) & \cong \mathbb{Z}_{2}[X, Y] /\left\langle X^{4}, u X^{2}+Y^{2}\right\rangle, \\
H^{*}\left(N\left(u^{\prime}\right) ; \mathbb{Z}_{2}\right) & \cong \mathbb{Z}_{2}[x, y] /\left\langle x^{4}, u^{\prime} x^{2}+x y+y^{2}\right\rangle .
\end{aligned}
$$

Now, the element $u X+Y$ in $H^{2}\left(M_{\alpha}(u) ; \mathbb{Z}_{2}\right)$ satisfies

$$
(u X+Y)^{2}=u^{2} X^{2}+2 u X Y+Y^{2} \equiv u X^{2}+Y^{2}(=0) \bmod 2 .
$$

However, the squares of all non-zero elements $x, y, x+y$ in $H^{2}\left(N\left(u^{\prime}\right) ; \mathbb{Z}_{2}\right)$ are not zero because of its ring structure. Hence

$$
H^{*}\left(M_{\alpha}(u)\right) \nRightarrow H^{*}\left(N\left(u^{\prime}\right)\right) \quad \text { for all } u, u^{\prime} \in \mathbb{Z} .
$$

Corollary 3.4 The classes $\mathcal{M}$ and $\mathcal{N}$ are disjoint. 


\section{Cohomological rigidity of $\mathcal{N}$}

In this section, we prove the cohomological rigidity of the class $\mathcal{N}$. It is enough to prove the following lemma.

Lemma 4.1 The following statements are equivalent for integers $u, u^{\prime}$ :

(1) $H^{*}(N(u)) \cong H^{*}\left(N\left(u^{\prime}\right)\right)$.

(2) $u=u^{\prime}$.

Proof Because (2) $\Rightarrow(1)$ is trivial, it is enough to show (1) $\Rightarrow(2)$. Assume there is an isomorphism $f: H^{*}(N(u)) \cong H^{*}\left(N\left(u^{\prime}\right)\right)$, where

$$
\begin{aligned}
& H^{*}(N(u)) \cong \mathbb{Z}[X, Y] /\left\langle X^{4}, u X^{2}+x y+Y^{2}\right\rangle, \\
& H^{*}\left(N\left(u^{\prime}\right)\right) \cong \mathbb{Z}[x, y] /\left\langle x^{4}, u^{\prime} x^{2}+x y+y^{2}\right\rangle .
\end{aligned}
$$

Here we may set

$$
f(X)=a x+b y \quad \text { and } \quad f(Y)=c x+d y
$$

for some $a, b, c, d \in \mathbb{Z}$ such that $a d-b c=\epsilon= \pm 1$. By taking its inverse, we also have

$$
f^{-1}(x)=d \epsilon X-b \epsilon Y \quad \text { and } \quad f^{-1}(y)=-c \epsilon X+a \epsilon Y .
$$

Because $f\left(Y^{2}+X Y+u X^{2}\right)=0$ and $f^{-1}\left(y^{2}+x y+u^{\prime} x^{2}\right)=0$, we get

$$
\begin{aligned}
& c^{2}-d^{2} u^{\prime}=-u a^{2}+b^{2} u u^{\prime}-a c+b d u^{\prime}, \\
& 2 c d-d^{2}=-2 a b u+b^{2} u-a d-b c+b d .
\end{aligned}
$$

Because $f\left(X^{4}\right)=0$ and $f^{-1}\left(x^{4}\right)=0$, one of the following holds:

(1) $b=0$.

(2) $b \neq 0$ and $4 a^{3}-6 a^{2} b+4 a b^{2}\left(1-u^{\prime}\right)+b^{3}\left(2 u^{\prime}-1\right)=-4 d^{3}-6 d^{2} b-4 d b^{2}(1-$ $u)+b^{3}(2 u-1)=0$.

If $b=0$, then $|a|=|d|=1$. Therefore, by (4), $2 c=d-a$; ie $c=0$ if $d=a$ or $c=-a$ if $d=-a$. Because $c^{2}-u^{\prime}=-u-a c$ by (3), we have that $u=u^{\prime}$.

Assume $b \neq 0$. Because $4 a^{3}-6 a^{2} b+4 a b^{2}\left(1-u^{\prime}\right)+b^{3}\left(2 u^{\prime}-1\right)=0, b$ is even. Therefore, since $a d-b c= \pm 1, a$ is odd. We note that the equation $4 a^{3}-6 a^{2} b+$ $4 a b^{2}\left(1-u^{\prime}\right)+b^{3}\left(2 u^{\prime}-1\right)=0$ can be written

$$
(2 a-b)\left(2 a^{2}-2 a b+b^{2}-2 b^{2} u^{\prime}\right)=0 .
$$

Because $a$ is odd and $b$ is even, the second factor is not zero; therefore

$$
b=2 a \text {. }
$$


Since $a d-b c= \pm 1$, we conclude $(a, b)= \pm(1,2)$. The same argument applied to the equation $-4 d^{3}-6 d^{2} b-4 d b^{2}(1-u)+b^{3}(2 u-1)=0$ shows that $-b=2 d$ and $(d, b)= \pm(-1,2)$. Therefore, $(a, b, d)$ must be either $(1,2,-1)$ or $(-1,-2,1)$. Then $c=0$ or -1 in the former case while $c=0$ or 1 in the latter because $a d-b c= \pm 1$. In any case, it follows from (3) that $u^{\prime}+u=4 u u^{\prime}$, an identity which holds only when $u=u^{\prime}=0$ since $u, u^{\prime} \in \mathbb{Z}$. This completes the case where $b \neq 0$.

Theorem 1.1 and Lemma 4.1 give the next theorem, which establishes Theorem 1.2(1).

Theorem 4.2 The following three statements are equivalent:

(1) $N(u)$ and $N\left(u^{\prime}\right)$ are diffeomorphic.

(2) The cohomology rings $H^{*}(N(u))$ and $H^{*}\left(N\left(u^{\prime}\right)\right)$ are isomorphic.

(3) $u=u^{\prime} \in \mathbb{Z}$.

In particular, the class $\mathcal{N}$ is cohomologically rigid.

\section{Cohomological non-rigidity of $\mathbb{C P}_{2}^{8}$}

Lemma 5.1 The following two statements are equivalent for integers $u, u^{\prime}$ :

(1) $H^{*}\left(M_{\alpha}(u)\right) \cong H^{*}\left(M_{\alpha^{\prime}}\left(u^{\prime}\right)\right)$, where $\alpha, \alpha^{\prime} \in\{0,1\}$.

(2) $u=u^{\prime}$.

Proof Because (2) $\Rightarrow$ (1) is trivial, it is enough to show (1) $\Rightarrow(2)$. Assume there is an isomorphism $f: H^{*}\left(M_{\alpha}(u)\right) \rightarrow H^{*}\left(M_{\alpha^{\prime}}\left(u^{\prime}\right)\right)$, where

$$
\begin{aligned}
H^{*}\left(M_{\alpha}(u)\right) & \cong \mathbb{Z}[X, Y] /\left\langle X^{4}, u X^{2}+Y^{2}\right\rangle, \\
H^{*}\left(M_{\alpha^{\prime}}\left(u^{\prime}\right)\right) & \cong \mathbb{Z}[x, y] /\left\langle x^{4}, u^{\prime} x^{2}+y^{2}\right\rangle .
\end{aligned}
$$

We may use the same representation for $f$ as in the proof of Lemma 4.1. Note that $f\left(u X^{2}+Y^{2}\right)=0$ and $f^{-1}\left(u^{\prime} x^{2}+y^{2}\right)=0$. Using the representation of $f$, we have

$$
\begin{aligned}
& u a^{2}-u u^{\prime} b^{2}+c^{2}-u^{\prime} d^{2}=0, \\
& u^{\prime} d^{2}-u u^{\prime} b^{2}+c^{2}-a^{2} u=0
\end{aligned}
$$

which lead to

$$
\begin{aligned}
c^{2} & =b^{2} u u^{\prime}, \\
u a^{2} & =u^{\prime} d^{2} .
\end{aligned}
$$


Because $X^{4}=0$,

$$
a b\left(a^{2}-b^{2} u^{\prime}\right)=0 .
$$

We first assume $a b \neq 0$. Then

$$
a^{2}=b^{2} u^{\prime}
$$

Together with (7) and (8), we have

$$
c^{2} b^{2}=b^{4} u u^{\prime}=b^{2} a^{2} u=b^{2} d^{2} u^{\prime}=a^{2} d^{2} .
$$

This implies

$$
(a d-b c)(a d+b c)=\epsilon(a d+b c)=0 .
$$

Hence $a d=-b c$. However this gives a contradiction because $a d-b c=2 a d=\epsilon= \pm 1$. Consequently, $a b=0$. Since $a d-b c=\epsilon$, if $a=0$ then $|b|=|c|=1$, so $u=u^{\prime}= \pm 1$ by (7), and if $b=0$ then $|a|=|d|=1$, so $u=u^{\prime}$ by (8). This establishes the lemma.

Lemma 5.1 says that cohomology rings of $\mathcal{M}$ are not affected by $\alpha \in \mathbb{Z}_{2}$. On the other hand, the goal of this section is to prove the following theorem, that some topological types of $\mathcal{M}$ are affected by $\alpha \in \mathbb{Z}_{2}$.

Theorem 5.2 Assume $u(u+1) / 12 \in \mathbb{Z}$ and $\alpha, \beta \in \mathbb{Z}_{2}$. The following are equivalent:

(1) $M_{\alpha}(u)$ and $M_{\beta}\left(u^{\prime}\right)$ are diffeomorphic.

(2) $(\alpha, u)=\left(\beta, u^{\prime}\right) \in \mathbb{Z}_{2} \times \mathbb{Z}$.

(3) $M_{\alpha}(u)$ and $M_{\beta}\left(u^{\prime}\right)$ are homotopy equivalent.

In order to prove Theorem 5.2, we first compute the 6-dimensional homotopy group of $M_{\alpha}(u)$ in Proposition 5.4. Now $M_{\alpha}(u)$ can be defined by the following pull-back diagram.

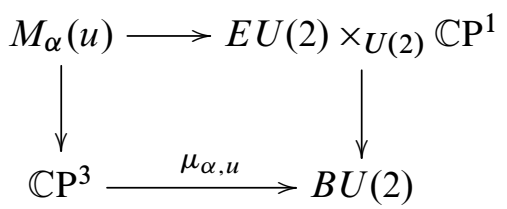

Let $p: S^{7} \rightarrow \mathbb{C P}^{3}$ be the canonical $S^{1}$-fibration and $P\left(\xi_{\alpha, u}\right)$ be the pull-back of $M_{\alpha}(u)$ along $p$. Namely, the following diagram commutes.

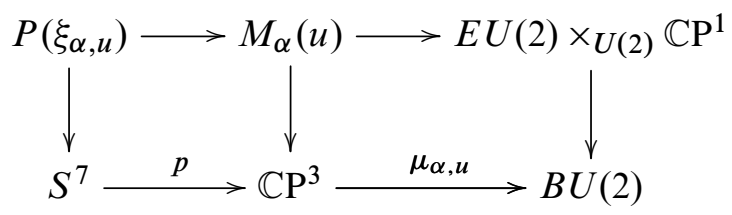


Lemma 5.3 For $* \geq 3, \pi_{*}\left(P\left(\xi_{\alpha, u}\right)\right) \cong \pi_{*}\left(M_{\alpha}(u)\right)$.

Proof Because $P\left(\xi_{\alpha, u}\right)$ is the pull-back of $M_{\alpha}(u)$, the homotopy exact sequences of $P\left(\xi_{\alpha, u}\right)$ and $M_{\alpha}(u)$ satisfy the following commutative diagram.

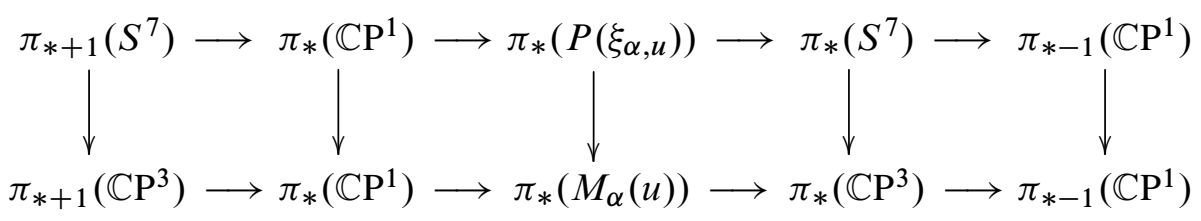

From the homotopy exact sequence of the fibration $S^{1} \rightarrow S^{7} \rightarrow \mathbb{C P}^{3}$, we have $\pi_{*}\left(S^{7}\right) \cong \pi_{*}\left(\mathbb{C P}^{3}\right)$ for $* \geq 3$. Therefore, by the five lemma, the proof is complete.

Proposition 5.4 Assume $u(u+1) / 12 \in \mathbb{Z}$.

(1) $\pi_{6}\left(P\left(\xi_{\alpha, u}\right)\right) \cong \pi_{6}\left(M_{\alpha}(u)\right) \cong \mathbb{Z}_{12}$ if $\alpha \equiv u(u+1) / 12(\bmod 2)$.

(2) $\pi_{6}\left(P\left(\xi_{\beta, u}\right)\right) \cong \pi_{6}\left(M_{\beta}(u)\right) \cong \mathbb{Z}_{6}$ if $\beta \not \equiv u(u+1) / 12(\bmod 2)$.

Proof First we prove (1). If $u(u+1) / 12 \in \mathbb{Z}$ and $\alpha \equiv u(u+1) / 12(\bmod 2)$, then it follows from [1] that $\xi_{\alpha, u}$ is induced from the rank-2 complex vector bundle over $\mathbb{C P}^{4}$. Namely, the following diagram commutes.

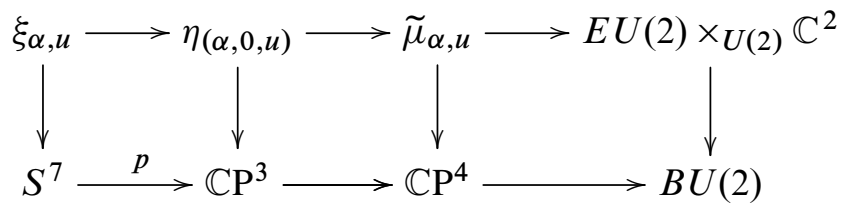

On the other hand, $\pi_{7}\left(\mathbb{C P}^{4}\right) \cong \pi_{7}\left(S^{9}\right)=\{0\}$, using the homotopy exact sequence for the fibration $S^{1} \rightarrow S^{9} \rightarrow \mathbb{C P}^{4}$. This implies that $\xi_{\alpha, u}$ is the trivial $\mathbb{C}^{2}$-bundle over $S^{7}$. Therefore,

$$
P\left(\xi_{\alpha, u}\right)=S^{7} \times \mathbb{C} P^{1}
$$

when $u(u+1) / 12 \in \mathbb{Z}$ and $\alpha \equiv u(u+1) / 12(\bmod 2)$. Hence, we also have

$$
\pi_{6}\left(M_{\alpha}(u)\right) \cong \pi_{6}\left(S^{7} \times \mathbb{C P}^{1}\right) \cong \pi_{6}\left(\mathbb{C P}^{1}\right) \cong \mathbb{Z}_{12} .
$$

Next we prove the second statement. Let $\mu_{\alpha, u}: \mathbb{C P}^{3} \rightarrow B U(2)$ be a continuous map which induces the above $\eta_{(\alpha, 0, u)}$, and $\beta$ be the element in $\mathbb{Z}_{2}$ which is not equal to $\alpha$. Let $x \in \mathbb{C P}^{3}$ and $s=\mu_{\alpha, u}(x) \in B U(2)$ be base points. Take a disk neighborhood around $x \in \mathbb{C P}^{3}$ and pinch its boundary to a point, ie the boundary of $D^{6} \subset \mathbb{C P}^{3}$ pinches to a point; then we obtain a surjective map

$$
\rho: \mathbb{C P} \rightarrow \mathbb{C P}^{3} \vee S^{6},
$$


where $\mathbb{C P}^{3} \vee S^{6}$ may be regarded as the wedge sum with respect to the base points $x \in \mathbb{C P}^{3}$ and $y \in S^{6}$. Due to Theorem 1.1, we have $\eta_{(\beta, 0, u)} \not \equiv \eta_{(\alpha, 0, u)}$. This implies that the vector bundle $\eta_{(\beta, 0, u)}$ is induced from the continuous map

$$
\mu_{\beta, u}: \mathbb{C} P^{3} \stackrel{\rho}{\longrightarrow} \mathbb{C} P^{3} \vee S^{6} \stackrel{v_{\alpha}}{\longrightarrow} B U(2),
$$

where $v_{\alpha}=\mu_{\alpha, u} \vee \kappa$ for the generator $\kappa \in \pi_{6}(B U(2), s) \cong \mathbb{Z}_{2} \cdot{ }^{1}$ Hence, we have the following commutative diagram.

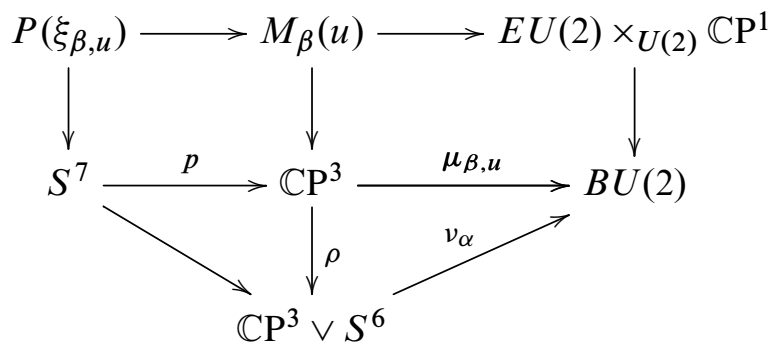

From the $\mathbb{C P}^{1}$-fibrations $\mathbb{C P}^{1} \rightarrow P\left(\xi_{\beta, u}\right) \rightarrow S^{7}$ and $\mathbb{C P}^{1} \rightarrow E U(2) \times_{U(2)} \mathbb{C P}^{1} \cong$ $B T^{2} \rightarrow B U(2)$ in the diagram (12), we get the following commutative diagram.

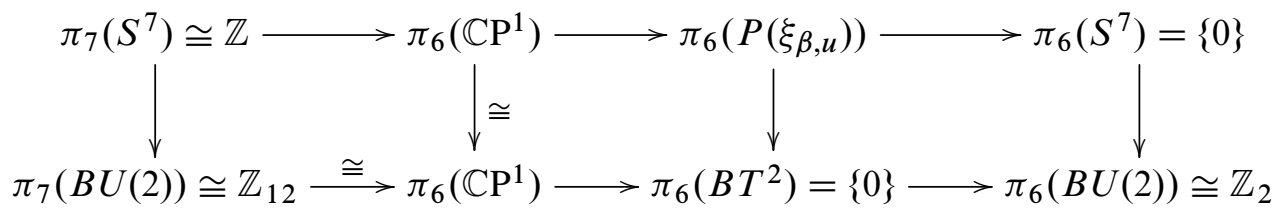

This diagram shows that the following sequence is exact:

$$
\mathbb{Z} \cong \pi_{7}\left(S^{7}\right) \rightarrow \pi_{7}(B U(2))\left(\cong \mathbb{Z}_{12}\right) \rightarrow \pi_{6}\left(P\left(\xi_{\beta, u}\right)\right) \rightarrow\{0\} .
$$

In this diagram, the left homomorphism is induced from $\tilde{\mu}:=\mu_{\beta, u} \circ p: S^{7} \rightarrow B U(2)$, say $\tilde{\mu}_{\#}: \mathbb{Z} \rightarrow \mathbb{Z}_{12}$. We claim $\tilde{\mu}_{\#}(1)=[6]_{12} \in \mathbb{Z}_{12}$. Because the diagram (12) is commutative, we can think of $\tilde{\mu}:=\mu_{\beta, u} \circ p: S^{7} \rightarrow B U(2)$ as being defined by passing through the map $v_{\alpha}: \mathbb{C P}^{3} \vee S^{6} \rightarrow B U(2)$; ie $\tilde{\mu}=v_{\alpha} \circ \rho \circ p$. Because $v_{\alpha}=\mu_{\alpha, u} \vee \kappa$, we also have

$$
\tilde{\mu}=\left(\mu_{\alpha, u} \vee \kappa\right) \circ \rho \circ p=\left(\mu_{\alpha, u} \circ \rho \circ p\right) \vee(\kappa \circ \rho \circ p) .
$$

By the argument we used while proving the first statement, we see that $\mu_{\alpha, u} \circ \rho \circ p$ induces the trivial bundle over $S^{7}$; ie $\mu_{\alpha, u} \circ \rho \circ p$ is homotopic to the trivial map. This

\footnotetext{
${ }^{1}$ This construction induces the free $\pi_{6}(B U(2)) \cong \pi_{5}(U(2)) \cong \mathbb{Z}_{2}$ action on $\widetilde{K S p}\left(\mathbb{C P}^{3}\right) \cong \mathbb{Z}_{2} \oplus \mathbb{Z} ;$ see [1].
} 
also implies that the decomposition

$$
\tilde{\mu}: S^{7} \stackrel{p}{\longrightarrow} \mathbb{C} P^{3} \stackrel{\rho}{\longrightarrow} \mathbb{C P}^{3} \vee S^{6} \stackrel{\pi}{\longrightarrow} S^{6} \stackrel{\kappa}{\longrightarrow} B U(2)
$$

exists up to homotopy, where $\pi$ is the collapsing map of $\mathbb{C P}^{3}$ to a point. Therefore, we have the following decomposition for the induced map:

$$
\tilde{\mu}_{\#}: \pi_{7}\left(S^{7}\right) \stackrel{\Psi_{\#}}{\longrightarrow} \pi_{7}\left(S^{6}\right) \cong \mathbb{Z}_{2} \stackrel{\kappa_{\sharp}}{\longrightarrow} \pi_{7}(B U(2)) \cong \mathbb{Z}_{12},
$$

where the first map is induced from the surjective map $\Psi=\pi \circ \rho \circ p$. Because $\Psi$ is surjective, ie not homotopic to the trivial map, we have $\Psi_{\#}(1)=[1]_{2}$ (the generator of $\left.\pi_{7}\left(S^{6}\right) \cong \mathbb{Z}_{2}\right)$. Moreover, because $\kappa \in \pi_{6}(B U(2)) \cong \mathbb{Z}_{2}$ is the generator, ie nontrivial map, we have $\kappa_{\#}\left([1]_{2}\right)=[6]_{12} \in \mathbb{Z}_{12}$. This shows that $\tilde{\mu}_{\#}(1)=[6]_{12}$; therefore $\tilde{\mu}_{\#}\left(\pi_{7}\left(S^{7}\right)\right)=\left\{[0]_{12},[6]_{12}\right\} \subset \mathbb{Z}_{12}$.

Consequently, by the exact sequence (13),

$$
\pi_{6}\left(P\left(\xi_{\beta, u}\right)\right) \cong \pi_{7}(B U(2)) / \tilde{\mu}_{\#}\left(\pi_{7}\left(S^{7}\right)\right) \cong \mathbb{Z}_{12} /\left\{[0]_{12},[6]_{12}\right\} \cong \mathbb{Z}_{6} .
$$

By Lemma 5.3, we have the statement.

Remark 2 For example, the condition $u(u+1) / 12 \in \mathbb{Z}$ is satisfied when $u=0$ and $u=3$. In these cases, using Proposition 5.4, we have

$$
\pi_{6}\left(M_{\alpha}(0)\right) \cong\left\{\begin{array} { l l } 
{ \mathbb { Z } _ { 1 2 } } & { \text { for } \alpha \equiv 0 } \\
{ \mathbb { Z } _ { 6 } } & { \text { for } \alpha \equiv 1 }
\end{array} \text { and } \quad \pi _ { 6 } ( M _ { \alpha } ( 3 ) ) \cong \left\{\begin{array}{ll}
\mathbb{Z}_{6} & \text { for } \alpha \equiv 0 \\
\mathbb{Z}_{12} & \text { for } \alpha \equiv 1 .
\end{array}\right.\right.
$$

On the other hand, the case when $u=1$ does not satisfy the condition $u(u+1) / 12 \in \mathbb{Z}$. It follows from the cohomology ring of the flag manifold of type $\mathrm{C}$ (see for example [2] or [6]) that the flag manifold $\mathrm{Sp}(2) / T^{2}$ is one for which $u=1$; ie $M_{0}(1)$ or $M_{1}(1)$. However, using the homotopy exact sequence for the fibration $T^{2} \rightarrow \operatorname{Sp}(2) \rightarrow \operatorname{Sp}(2) / T^{2}$ and the computation in [8],

$$
\pi_{6}\left(\operatorname{Sp}(2) / T^{2}\right) \cong \pi_{6}(\operatorname{Sp}(2))=0 .
$$

Therefore, Proposition 5.4 is not true in the case where $u(u+1) / 12 \notin \mathbb{Z}$.

Proof of Theorem 5.2 (2) $\Rightarrow$ (1) is trivial, as is (1) $\Rightarrow$ (3). We claim (3) $\Rightarrow$ (2). Assume $M_{\alpha}(u)$ and $M_{\beta}\left(u^{\prime}\right)$ are homotopy equivalent. Then $H^{*}\left(M_{\alpha}(u)\right) \cong H^{*}\left(M_{\beta}\left(u^{\prime}\right)\right)$. Therefore, it follows from Lemma 5.1 that $u=u^{\prime}$. Moreover, in this case, $\pi_{6}\left(M_{\alpha}(u)\right) \cong$ $\pi_{6}\left(M_{\beta}(u)\right)$. If $\alpha \neq \equiv \bmod 2$, then this gives a contradiction to Proposition 5.4. Hence, $\alpha \equiv \beta \bmod 2$. We have (3) $\Rightarrow$ (2). This establishes Theorem 5.2.

Lemma 5.1 and Theorem 5.2 imply the following corollary, establishing Theorem 1.2(2).

Corollary 5.5 The set of 8-dimensional $\mathbb{C P}$ manifolds is not cohomologically rigid. 
Note that if we restrict the class of 8 -dimensional $\mathbb{C P}$ manifolds to the 8-dimensional generalized Bott manifolds with height 2, then cohomological rigidity holds [4]. On the other hand, the following seems to be more natural to ask of the class of $\mathbb{C P}$ manifolds $\mathbb{C P M}$ than the cohomological rigidity problem.

Problem 2 Is the class $\mathbb{C P} \mathcal{M}$ of $\mathbb{C P}$ manifolds (up to diffeomorphism) determined by homotopy types? More precisely, are $M_{1}, M_{2} \in \mathbb{C P M}$ diffeomorphic if they have the same homotopy type?

\section{References}

[1] MF Atiyah, E Rees, Vector bundles on projective 3-space, Invent. Math. 35 (1976) 131-153 MR0419852

[2] A Borel, Sur la cohomologie des espaces fibrés principaux et des espaces homogènes de groupes de Lie compacts, Ann. of Math. 57 (1953) 115-207 MR0051508

[3] V M Buchstaber, T E Panov, Torus actions and their applications in topology and combinatorics, University Lecture Series 24, Amer. Math. Soc. (2002) MR1897064

[4] S Choi, M Masuda, D Y Suh, Topological classification of generalized Bott towers, Trans. Amer. Math. Soc. 362 (2010) 1097-1112 MR2551516

[5] S Choi, M Masuda, D Y Suh, Rigidity problems in toric topology: A survey, Tr. Mat. Inst. Steklova 275 (2011) 188-201 MR2962979

[6] Y Fukukawa, H Ishida, M Masuda, The cohomology ring of the GKM graph of a flag manifold of classical type, Kyoto J. Math. 54 (2014) 653-677 MR3263556

[7] S Kuroki, D Y Suh, Complex projective towers and their cohomological rigidity up to dimension six arXiv:1203.4403 to appear in Proc. Steklov Inst. Math.

[8] M Mimura, H Toda, Homotopy groups of SU(3), SU(4) and Sp(2), J. Math. Kyoto Univ. 3 (1963) 217-250 MR0169242

Graduate School of Mathematical Sciences, University of Tokyo 3-8-1 Komaba Meguro-ku, Tokyo 153-8914, Japan

Department of Mathematical Sciences, KAIST 335 Gwahangno, Yuseong Gu, Daejeon 305-701, South Korea kuroki@ms.u-tokyo.ac.jp, dysuh@math.kaist.ac.kr http://www.ms.u-tokyo.ac.jp/ kuroki/

Received: 30 November 2013 Revised: 25 July 2014 